\title{
Optimal Control of DC-DC Converter using Mixed Logical Dynamical System Model
}

\author{
Nobuhiro AsanoＳtudent Member (Mie University, asano@cs.elec.mie-u.ac.jp) \\ Tadanao Zanma Member (Mie University, zanma@elec.mie-u.ac.jp) \\ Muneaki Ishida Member (Mie University, ishida@elec.mie-u.ac.jp)
}

Keywords: dc-dc converter, hybrid dynamical systems, powerelectronics, model predictive control and multi-parametric mixed integer quadratic programming

Recently, control systems with switching of control modes and the discrete input, which is called hybrid dynamical systems, are actively researched. The hybrid dynamical systems consist of different types of dynamical systems, that is continuous time systems and the discrete event systems. Generally, the continuous time system is expressed by differential or difference equations. On the other hand, the discrete event system is described by the logic or state machines such as automata. If a system can be regarded as the hybrid dynamical system, both continuous behaviors and discrete events can be dealt with concurrently. Therefore, the hybrid dynamical system can model many systems as a single model without dividing into two systems.

Systems containing power electronic circuits include both the continuous and discontinuous behaviors. The continuous time behavior like current or voltage is yielded by the electric circuit by passive elements whereas the discontinuous one is on-off signal given to switching devices like MOSFET and/or IGBT. Thus, such a system is typical example of the hybrid dynamical system.

For design problem of the hybrid dynamical system, a variety of approaches have been proposed. Especially, modeling and synthesis based on mixed logical dynamical (MLD) systems by Bemporad et al. has much potential in the synthesis since the formulation is similar to the linear discrete time state space representation. The solution of the design is obtained by solving the optimization problem with help of model predictive control (MPC). Specifically, the problem is solved as a mixed-integer quadratic programming (MIQP) problem. The method is expected that it can generate better control performance than that by the conventional method if applied to the output control of the power converter.

However, it is difficult to solve the optimal problem online due to computation burden as the control period is considerably short in power converters compared to mechanical systems.

This paper proposes an output control method using the MPC for the output control problem of the dc-dc converter. The considered system is described as a MLD system. The control should achieve
2 quick tracking to the reference in transient state while the minimum switching frequency in steady state. The control input is obtained by calculation of the optimization problem which is solved by means of MPC.

To verify the effectiveness of the proposed method, some numerical simulations compared online with offline are illustrated.

Fig. 1 depeicts the state space partition. Fig. 1 is obtained off-line. Fig. 2 is the simulation result using the state space partition. It can be seen that the output is tracked the reference.

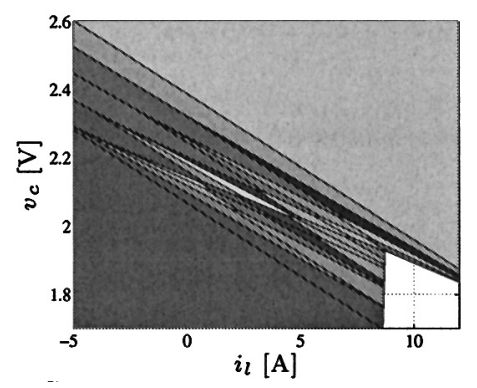

Fig. 1. State partition closeup (active mode)

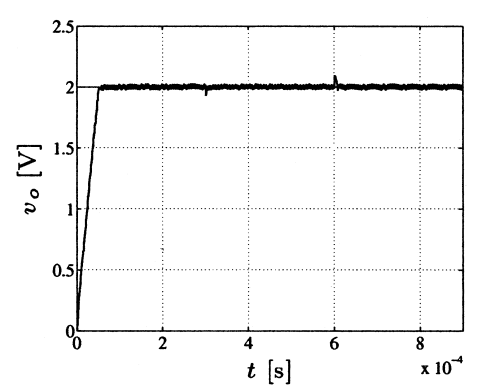

Fig. 2. Output voltage $v_{o}$ 


\title{
Optimal Control of DC-DC Converter using Mixed Logical Dynamical System Model
}

\author{
Nobuhiro Asano* Student Member \\ Tadanao Zanma* Member \\ Muneaki Ishida* Member
}

This paper presents an optimal control method of dc-dc converters. The method is based on hybrid dynamical system theory. Specifically, the input of the considered system is confined to discrete value whereas the output to be controlled is continuous value. The system is modeled as a mixed logical dynamical system. Then, the optimal control law is obtained by means of model predictive control. The effectiveness of the proposed approach is illustrated through some numerical simulations.

Keywords: dc-dc converter, hybrid dynamical systems, powerelectronics, model predictive control and multi-parametric mixed integer quadratic programming

\section{Introduction}

Recently, control systems with switching of control modes and the discrete input, which is called hybrid dynamical systems, are actively researched ${ }^{(1)-(3)}$. The hybrid dynamical systems consist of different types of dynamical systems, that is continuous time systems and the discrete event systems. Generally, the continuous time system is expressed by differential or difference equations while the discrete event system is described by logics or state machines such as automata. If a system can be regarded as the hybrid dynamical system, both continuous behaviors and discrete events can be dealt with concurrently. Therefore, the hybrid dynamical system can model many systems as a single model without dividing into two systems.

Systems containing power electronic circuits include both the continuous and discontinuous behaviors. The continuous behavior like current or voltage is yielded by the electric circuit by passive elements whereas the discontinuous one is on-off signal given to switching devices like MOSFET and/or IGBT. Thus, such a system is typical example of the hybrid dynamical system. In addition, recent developments of DSP and FPGA are expected to propel the digital control in power electronic circuits.

For design problem of the hybrid dynamical system, a variety of approaches have been proposed. Especially, modeling and synthesis based on mixed logical dynamical (MLD) systems by Bemporad et al. has much potential in the synthesis since the formulation is similar to the linear discrete time state space representation ${ }^{(7)}$. The solution of the design is obtained by solving the optimization problem with help of model predictive control (MPC) ${ }^{(4)(5)}$. Specifically, the problem is solved as a mixed-integer linear or quadratic programming (MILP or MIQP) problem. It is expected that the

\footnotetext{
* Faculty of Engineering, Mie University,

1577, Kurimamachiya, Tsu, Mie 514-8507
}

method can achieve better control performance than that by conventional methods if applied to the output control of the power converter. However, it is difficult to solve the optimal problem online due to computation burden since the control period is considerably short in power converters compared to mechanical systems.

This paper proposes a control method using the MPC for the output control problem of the dc-dc converter ${ }^{\left({ }^{(9)}\right.}{ }^{(9)}$. The considered system is described as an MLD system. In the work ${ }^{(9)}$, one control period is divided into $N$ submodels. Thus, further auxiliary variables are needed. In addition, the state variable among the submodels is handled as some kind of averaged one. On the contrary, this paper requires no averaging method. The explicit switching law is given as direct gate signal for the switching devices. Moreover, it is emphasized that the quadratic objective function not addressed in the work ${ }^{(9)}$ is addressed in this paper so that not only the tracking error but also the switching loss can be taken into account. The control should achieve quick tracking to the reference in transient state while the minimum switching frequency in steady state. The control input is obtained by calculation of the optimization problem which is solved by means of MPC. To verify the effectiveness of the proposed method, some numerical simulations compared online with offline are illustrated.

This paper is organized as follows. In Section 2, a stepdown dc-dc converter and MLD are introduced. After that the optimization problems are described. In Section 3, an optimal control method is proposed. In the method, formulation of constraints and transformation to mp-MIQP are described. The effectiveness of the proposed method is shown through some simulations in Section 4. Finally, Section 5 concludes this paper.

\section{Preliminaries}

In this section, a step-down dc-dc converter is considered as the plant to be controlled. After the formulation, 


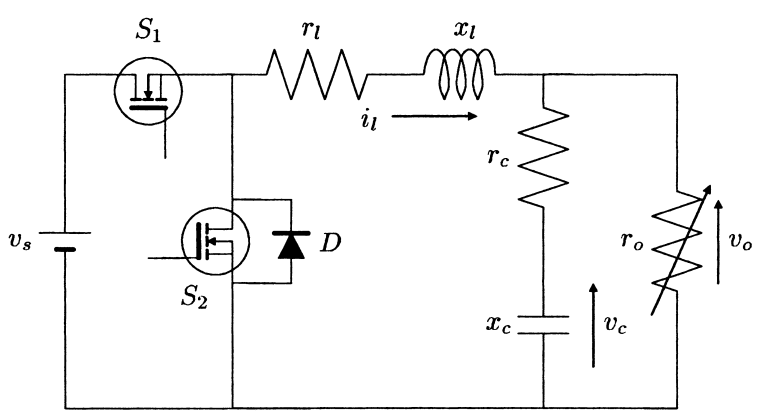

Fig. 1. Topology of the step-down dc-dc converter

MLD system, MPC, MIQP and multi-parametric MIQP (mpMIQP) are reviewed.

2.1 Step-down Dc-Dc Converter A circuit topology of the step-down dc-dc converter is shown in Fig. 1. The dcdc converter controls the load voltage $v_{o}$ by on or off of the switch. The resistance $r_{o}$ expresses the application driven by the dc-dc converter equivalently. The Equivalent Series Resistance (ESR) of the capacitor and the internal resistance of the inductor are denoted by $r_{c}$ and $r_{l}$, respectively while $x_{l}$ and $x_{c}$ represent the inductance and the capacitance of the low-pass filtering stage, respectively. The switches $S_{1}$ and $S_{2}$ can not be conducted simultaneously. Together with the diode $D$, the switch $S_{2}$ provides a path for the inductor current $i_{l}$ regardless whether it is positive or negative. In this paper, the dc-dc converter shown in Fig. 1 is the intended system. Continuous time state-space representation of the system is given as follows,

$$
\begin{aligned}
\dot{x}(t) & =A_{c} x(t)+B_{c} u(t) \\
y(t) & =C_{c} x(t) \cdots \cdots
\end{aligned}
$$

where $x(t)=\left[\begin{array}{ll}i_{l}(t) & v_{c}(t)\end{array}\right]^{\prime}$. Denoted by $i_{l}(t)$ and $v_{c}(t)$ are the inductor current and the capacitor voltage, respectively. The matrices $A_{c}, B_{c}$ and $C_{c}$ are given by

$$
\begin{aligned}
& A_{c}=\left[\begin{array}{cc}
-\frac{1}{x_{l}}\left(r_{l}+\frac{r_{o} r_{c}}{r_{o}+r_{c}}\right) & -\frac{1}{x_{l}}\left(\frac{r_{o}}{r_{o}+r_{c}}\right) \\
\frac{1}{x_{c}}\left(\frac{r_{o}}{r_{o}+r_{c}}\right) & -\frac{1}{x_{c}}\left(\frac{1}{r_{o}+r_{c}}\right)
\end{array}\right], \\
& B_{c}=\left[\begin{array}{c}
\frac{1}{x_{l}} \\
0
\end{array}\right]
\end{aligned}
$$

and $C_{c}=\left[\begin{array}{ll}\frac{r_{o} r_{c}}{r_{o}+r_{c}} & \frac{r_{o}}{r_{o}+r_{c}}\end{array}\right]$, respectively. Eqs. (1) and (2) are sampled by $T_{s}$. The discrete time is described anew as $t$. Thus, the considered system is recast as follows,

$$
\begin{aligned}
& x(t+1)=A x(t)+B u(t), \\
& y(t)=C x(t), \cdots \cdots \cdots
\end{aligned}
$$

where $A=e^{A_{c} T_{s}}, B=\int_{0}^{T_{s}} e^{A_{c} \tau} d \tau B_{c}$ and $C=C_{c}$. Note that the value of input is limited to either 0 or $v_{s}$, which can be rewritten as follows,

$$
(\forall t) u(t) \in\left\{0, v_{s}\right\}
$$

2.2 MLD System ${ }^{(7)} \quad$ The MLD systems are described by linear dynamic equations subject to linear mixed-integer inequalities. Discrete events included in process are represented using logic variables. One of the advantages is in that
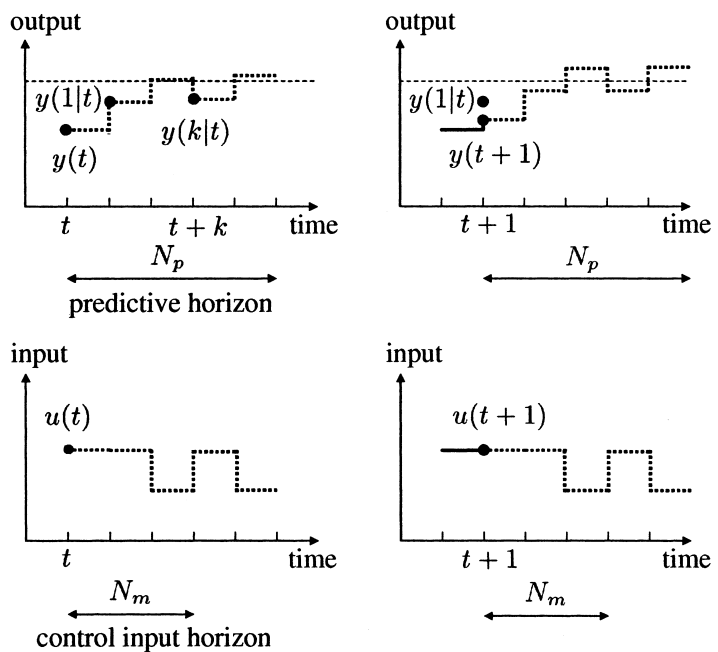

Fig. 2. Outline of MPC

the logical formula can be described linear inequalities and MPC can be applied to the MLD system.

The general MLD form is given as follows,

$$
\begin{aligned}
& x(t+1)=A x(t)+B_{1} u(t)+B_{2} \delta(t)+B_{3} z(t) \cdots \cdots \\
& y(t)=C x(t)+D_{1} u(t)+D_{2} \delta(t)+D_{3} z(t) \cdots \cdots \cdots \\
& E_{2} \delta(t)+E_{3} z(t) \leq E_{1} u(t)+E_{4} x(t)+E_{5} \cdots \cdots \cdots
\end{aligned}
$$

where $x(t), u(t)$ and $y(t)$ are states, inputs and outputs as is the usual linear systems, respectively. Additionally, $\delta(t)$ and $z(t)$ are introduced as binary and auxiliary continuous variables, respectively. $A, B_{i}, C, D_{i}$ and $E_{i}$ are appropriate matrices. As a result, Eqs. (6), (7) and (8) consist of the usual linear discrete time state space representation with the auxiliary variables of $\delta$ and $z$ and the set of linear inequalities which represents the constraints on the system. Eq. (8) is also used to determine $\delta$ or $z$ uniquely. Thus, the hybrid dynamical systems can be represented a familiar formulation and inequalities by the MLD system form.

2.3 MPC Fig. 2 shows the outline of the MPC. Suppose that output $y(t)$ is observed at $t$. Then, $y(k \mid t)$ is calculated by the use of the model, where $y(k \mid t)$ denotes the predicted output at $t+k$. The input sequence $u(0 \mid t), u(1 \mid t), \ldots, u\left(N_{m}-\right.$ $1 \mid t)$ over control input horizon $\left[t, t+N_{m}\right]$ is determined so that the predicted trajectory approaches the reference for predictive horizon $\left[t+1, t+N_{p}\right] . N_{m}$ satisfies $N_{m} \leq N_{p}$. Only $u(t)=u(0 \mid t)$, the first term of the input sequence, is actually input to the plant and hold until $t+1$. When $y(t+1)$ is observed at $t+1$, the input is similarly determined by sliding the predicted horizon to one step ahead. Then, the procedure is repeated. In this paper, $N_{m}$ is simply set $N_{p}=N_{m}$.

2.4 MIQP and Mp-MIQP ${ }^{(6)} \quad$ The MIQP derives the values which minimize an estimation of a given objective function under constraints given by inequalities and/or equalities concerning integer variables. The general MIQP form is given as follows,

$$
\begin{aligned}
& \min _{v_{t}} v_{t}^{\prime} S_{1} v_{t}+2\left(S_{2}+x(t)^{\prime} S_{3}\right) v_{t} \\
& \text { subject to } F_{1} v_{t} \leq F_{2}+F_{3} x(t),
\end{aligned}
$$

where $v_{t}$ is 


$$
\begin{aligned}
& v_{t}=\left[\begin{array}{lll}
\Omega_{t}^{\prime} & \Delta_{t}^{\prime} & \Xi_{t}^{\prime}
\end{array}\right]^{\prime}, \ldots \ldots \ldots \ldots \\
& \Omega_{t}=\left[\begin{array}{lll}
u(0 \mid t) & \ldots & u\left(N_{p}-1 \mid t\right)
\end{array}\right]^{\prime}, \\
& \Delta_{t}=\left[\begin{array}{lll}
\delta(0 \mid t) & \ldots & \delta\left(N_{p}-1 \mid t\right)
\end{array}\right]^{\prime}, \\
& \Xi_{t}=\left[\begin{array}{lll}
z(0 \mid t) & \ldots & z\left(N_{p}-1 \mid t\right)
\end{array}\right]^{\prime} .
\end{aligned}
$$

The mp-MIQP is a kind of MIQP parameterized by multiple parameters. The MIQP in the MPC often appears as the mp-MIQP. In such a case, the mp-MIQP parameterized by the state $x$ of the model is described as follows,

$$
\begin{aligned}
& \min _{v} v^{\prime} H v+2 x^{\prime} F v+x^{\prime} Y x+2 C_{f} v+2 C_{x} x, \cdots \cdots \\
& \text { subject to } G v \leq W+E x, \cdots \cdots \cdots \cdots \cdots \cdots \cdots \cdots \cdots \cdots
\end{aligned}
$$

where $v$ is

$$
\begin{aligned}
& v=\left[\begin{array}{lll}
\Omega^{\prime} & \Delta^{\prime} & \Xi^{\prime}
\end{array}\right]^{\prime}, \ldots \\
& \Omega=\left[\begin{array}{lll}
u_{0} & \ldots & u_{N_{p}-1}
\end{array}\right]^{\prime}, \\
& \Delta=\left[\begin{array}{lll}
\delta_{0} & \ldots & \delta_{N_{p}-1}
\end{array}\right]^{\prime}, \\
& \Xi=\left[\begin{array}{lll}
z_{0} & \ldots & z_{N_{p}-1}
\end{array}\right]^{\prime} .
\end{aligned}
$$

Note that there exists a clear difference between notations of $v_{t}$ and $v$. The former is utilized for MIQP while the latter is for $\mathrm{mp}-\mathrm{MIQP}$.

If solved, the optimal solution of the mp-MIQP is given as the piece-wise affine state feedback form. Namely, the control law of explicit form parameterized by the state $x$ is obtained as follows,

$$
v=K_{i} x+h_{i} \text { if } x \in X_{i}, \cdots
$$

where $X_{i}(i=1,2, \ldots)$ are regions partitioned in the state space, $K_{i}$ and $h_{i}$ are the corresponding constant matrices and vectors. As Eq. (21) is available offline, the optimal input is determined online according to measured state at each sampling.

\section{Optimal Output Control Method of Dc-Dc Converter}

In this section, a problem that the carrier frequency can not be set so high in Pulse Width Modulation (PWM) is described. The system to be controlled is modeled as the hybrid dynamical system. For the system, a model predictive control (MPC) based control method is proposed.

3.1 Conventional Method The basic method which is currently used for the control of dc-dc converters is the PWM with the triangular wave. The PWM, which determines on-off switching timing, controls the average output voltage. It employs relatively high carrier frequency so that the output voltage tracks to the voltage reference. Under such a condition, the voltage reference at the rising edge and that at the half period of the triangular wave can be assumed to be almost equal. Thus, the average voltage in the time can approximate the voltage reference. However, the reference may vary in the half period of triangular wave carrier if the carrier frequency is lowered to decrease switching loss for the energy saving Therefore, the average voltage can not approximate the voltage reference any longer. One of possible reasons is in that the control frequency is determined by the carrier frequency only. Other reason may be in that the PWM focuses on only the average output characteristic and excludes switching behaviors.

In this section, the method based on the MPC for the output control is proposed, regarding the power converter as the hybrid dynamical system. It derives the optimal input by predicting controlled variables using the mathematical plant model and minimizing a given objective function.

3.2 Representation by MLD System The dc-dc converter is rewritten to the MLD system representation to obtain the plant model. As shown by Eqs. (3), (4) and (5), the output which is a continuous variable is controlled by the discrete input for the dc-dc converter.

The auxiliary $\delta$ of $0-1$ variable is introduced as a new input variable to describe the discrete variable. The variable is associated with as follows,

$$
\begin{aligned}
& {[\delta(t)=1] \rightarrow\left[z(t)=v_{s}\right],} \\
& {[\delta(t)=0] \rightarrow[z(t)=0],}
\end{aligned}
$$

where $z(t)$ is,

$$
0 \leq z(t) \leq v_{s}
$$

Note that $z(t)$ is a seeming continuous auxiliary variable. Eqs. (22) and (23) indicate that $z(t)=v_{s}$ if $\delta(t)=1$ whereas $z(t)=0$, otherwise. By replacing Eqs. (22) and (23) by their equivalent linear inequalities, the MLD system representation can be described as follows,

$$
\begin{aligned}
& x(t+1)=A x(t)+B z(t), \cdots \\
& y(t)=C x(t), \cdots \cdots \cdots \cdots \cdots \\
& {\left[\begin{array}{c}
1 \\
-1 \\
1 \\
-1
\end{array}\right] z(t) \leq\left[\begin{array}{c}
0 \\
-v_{s} \\
v_{s} \\
0
\end{array}\right] \delta(t)+\left[\begin{array}{c}
v_{s} \\
0 \\
0 \\
0
\end{array}\right] .}
\end{aligned}
$$

Inequality (27) reflects that $z(t)=v_{s}$ if $\delta(t)=1$ whereas $z(t)=0$ if $\delta(t)=0$. Namely, $\delta(t)$ can be considered as the state of the switch: $\delta(t)=1$ if the switch is on, $\delta(t)=0$ if it is off.

3.3 Output Control Method by MIQP To derive the input sequence by applying the MPC for the plant model given by the MLD system form, the objective function is set as follows,

$$
J\left(x(t), \Delta_{t}, \Xi_{t}\right)=\sum_{k=1}^{N_{p}}\left\|y(k \mid t)-v_{\text {ref }}\right\|_{2}^{2}+\Delta_{t}^{\prime} \tilde{H} \Delta_{t}+2 L \Delta_{t},
$$

where $v_{\text {ref }}$ denotes the voltage reference. Eq. (28) is rewritten as the general MIQP form like Eqs. (9) and (10) to solve the minimization problem. By using Eqs. (3) and (4), $y(k \mid t)$ is described as follows,

$$
\begin{aligned}
y(k \mid t) & =C\left(A^{k} x(t)+\sum_{j=0}^{k-1} A^{k-j-1} B z(j)\right) \\
& =C\left(A^{k} x(t)+G_{k} \Xi_{k}^{\prime}\right), \cdots \ldots \ldots
\end{aligned}
$$

where $G_{k}=\left[\begin{array}{llll}A^{k-1} B & A^{k-2} B & \ldots & B\end{array}\right]$. By substituting Eq. (29) into Eq. (28), Eq. (28) is given as follows, 


$$
\begin{aligned}
& J\left(x(t), \Delta_{t}, \Xi_{t}\right) \\
= & \sum_{k=1}^{N_{p}} \Xi_{t}^{\prime} G_{k}^{\prime} C^{\prime} C G_{k} \Xi_{t}-2 \sum_{k=1}^{N_{p}} v_{\mathrm{ref}}^{\prime} C G_{k} \Xi_{t} \\
& +2 \sum_{k=1}^{N_{p}} x(t)^{\prime} A^{\prime k} C^{\prime} C G_{k} \Xi_{t}+\Delta_{t}^{\prime} \tilde{H} \Delta_{t}+2 L \Delta_{t} .
\end{aligned}
$$

Connected with Eq. (9), the optimization problem of Eq. (30) is

$$
\min _{\Delta_{t}, \Xi_{t}}\left[\begin{array}{c}
\Delta_{t} \\
\Xi_{t}
\end{array}\right]^{\prime} S_{1}\left[\begin{array}{l}
\Delta_{t} \\
\Xi_{t}
\end{array}\right]+2\left(S_{2}+x(t)^{\prime} S_{3}\right)\left[\begin{array}{c}
\Delta_{t} \\
\Xi_{t}
\end{array}\right],
$$

where $S_{1}, S_{2}$ and $S_{3}$ are, respectively,

$$
\begin{aligned}
& S_{1}=\left[\begin{array}{cc}
\tilde{H} & O \\
O & \sum_{k=1}^{N_{p}} G_{k}^{\prime} C^{\prime} C G_{k}
\end{array}\right] \in \mathbb{R}^{2 N_{p} \times 2 N_{p}}, \\
& S_{2}=\left[\begin{array}{cc}
L & -\sum_{k=1}^{N_{p}} v_{\mathrm{ref}}^{\prime} C G_{k}
\end{array}\right] \in \mathbb{R}^{1 \times 2 N_{p}}, \cdots \\
& S_{3}=\left[\begin{array}{ll}
O & \sum_{k=1}^{N_{p}} A_{k}^{\prime} C^{\prime} C G_{k}
\end{array}\right] \in \mathbb{R}^{2 \times 2 N_{p}} . \cdots
\end{aligned}
$$

In this paper, $\tilde{H}$ and $L$ are zeros.

Let us rewrite the constraints as the general form like inequality (10). Recall that only two discrete input is permitted in the considered system. Such a constraint can be represented as follows, associating Eq. (27) with Eq. (10),

$$
\tilde{F}_{1}\left[\begin{array}{l}
\Delta_{t} \\
\Xi_{t}
\end{array}\right] \leq \tilde{F}_{2}+\tilde{F}_{3} x(t)
$$

where $\tilde{F}_{1}, \tilde{F}_{2}$ and $\tilde{F}_{3}$ are, respectively,

$$
\begin{aligned}
& \tilde{F}_{1}=\left[\begin{array}{cccccc}
0 & & & 1 & & \\
v_{s} & & O & -1 & & O \\
-v_{s} & & & 1 & & \\
0 & & & -1 & & \\
& \ddots & & & \ddots & \\
& & 0 & & & 1 \\
O & & v_{s} & O & & -1 \\
& & -v_{s} & & & 1 \\
& & & & & -1
\end{array}\right] \in \mathbb{R}^{4 N_{p} \times 2 N_{p}}, \\
& \tilde{F}_{2}=\left[\begin{array}{c}
v_{s} \\
0 \\
0 \\
0 \\
\vdots \\
v_{s} \\
0 \\
0 \\
0
\end{array}\right] \in \mathbb{R}^{4 N_{p}}, \tilde{F}_{3}=\left[\begin{array}{cc}
0 & 0 \\
0 & 0 \\
0 & 0 \\
0 & 0 \\
\vdots & \vdots \\
0 & 0 \\
0 & 0 \\
0 & 0 \\
0 & 0
\end{array}\right] \in \mathbb{R}^{4 N_{p} \times 2}
\end{aligned}
$$

Constraints of the inductor current limitation are added to avoid the switching device being broken due to the overcurrent to the inductance. More specifically, if the predictive inductor current at $t+1$, i.e. $i_{l}(1 \mid t)$, exceeds the limited value, $i_{l, \max }$, then the switch is forced to be off. Such an additional condition can be described as follows,

$$
\left[i_{l}(1 \mid t)>i_{l, \max }\right] \rightarrow[\delta(0)=0] .
$$

Transformed into the inequality, Eq. (37) is described as,

$$
i_{l}(1 \mid t)-i_{l, \max } \leq M(1-\delta(0)),
$$

where $M$ is the upper limit of $i_{l}$. As $x=\left[\begin{array}{ll}i_{l} & v_{c}\end{array}\right]^{\prime}$, replaced the first row of $A$ and the first element of $B$ with $A_{1}$ and $b_{1}$, respectively, $i_{l}(1 \mid t)$ is recast in,

$$
i_{l}(1 \mid t)=A_{1} x(t)+b_{1} z(0) .
$$

Consequently, by Eq. (39), inequality (38) has the following form,

$$
M \delta(0)+b_{1} z(0) \leq\left(M+i_{l, \max }\right)-A_{1} x(t) .
$$

Add Eq. (40) as new constraints to the last row of Eq. (36), then Eq. (36) is modified as follows,

$$
\begin{aligned}
& F_{1}=\left[\begin{array}{llllllll}
M & 0 & \cdots & 0 & b_{1} & 0 & \cdots & 0
\end{array}\right], \\
& F_{2}=\left[\begin{array}{c}
\tilde{F}_{2} \\
M+\tilde{i}_{l, \max }
\end{array}\right], F_{3}=\left[\begin{array}{c}
\tilde{F}_{3} \\
A_{1}
\end{array}\right] \text {. }
\end{aligned}
$$

If the objective function is described the general MIQP form as above, the optimal input can be derived. However, the optimization problem of the objective function of which its computation amount is so much should be solved every control period. Therefore, it is difficult to apply to the system with short control frequency like electrical circuits, especially, dc-dc converter. Consequently, the method is desired which solves the optimization problem by not online but offline and utilizes the result for the control. Such a method is realized by solving the optimization problem as mp-MIQP.

3.4 Output Control Method by Mp-MIQP Eq. (28) is adopted as the objective function. Multiplied out, Eq. (28) is described as follows,

$$
\begin{aligned}
& J(x, \Delta, \Xi) \\
= & \sum_{k=1}^{N_{p}} \Xi^{\prime} G_{k}^{\prime} C^{\prime} C G_{k} \Xi+2 \sum_{k=1}^{N_{p}} x^{\prime} A^{\prime k} C^{\prime} C G_{k} \Xi \\
& +\sum_{k=1}^{N_{p}} x^{\prime} A^{\prime k} C^{\prime} C A^{k} x-2 \sum_{k=1}^{N_{p}} v_{\mathrm{ref}}^{\prime} C G_{k} \Xi \\
& -2 \sum_{k=1}^{N_{p}} v_{\mathrm{ref}}^{\prime} C A^{k} x+\Delta^{\prime} \tilde{H} \Delta+2 L \Delta . \cdots \cdots \cdot
\end{aligned}
$$

Associated with Eq.(15), the optimization problem of Eq. (42) is transformed as follows,

$$
\begin{aligned}
\min _{\Delta, \Xi} & {\left[\begin{array}{l}
\Delta \\
\Xi
\end{array}\right]^{\prime} H\left[\begin{array}{l}
\Delta \\
\Xi
\end{array}\right]+2 x^{\prime} F\left[\begin{array}{l}
\Delta \\
\Xi
\end{array}\right]+x^{\prime} Y x } \\
& +2 C_{f}\left[\begin{array}{l}
\Delta \\
\Xi
\end{array}\right]+2 C_{x} x, \ldots \ldots \ldots .
\end{aligned}
$$

where $H, F$ and $C_{f}$ are $H=S_{1}, F=S_{3}$ and $C_{f}=S_{2}$, respectively. The others are, 


$$
\begin{aligned}
& Y=\sum_{k=1}^{N_{p}} A^{\prime k} C^{\prime} C A^{k}, \\
& C_{x}=-\sum_{k=1}^{N_{p}} v_{\text {ref }}^{\prime} C A^{k} .
\end{aligned}
$$

The constraints are given as

$$
G\left[\begin{array}{l}
\Delta \\
\Xi
\end{array}\right] \leq W+E x
$$

where $G, W$ and $E$ are $G=F_{1}, W=F_{2}$ and $E=F_{3}$, respectively. Transformed as above, the optimization problem is solved as mp-MIQP offline. Then, the result is employed to control.

\section{Numerical Simulation}

In this section, the effectiveness of the method proposed in the previous section is shown by applying it to the output control of dc-dc converter shown in Fig. 2. The specification is to achieve quick tracking to the reference in transient state with minimal switching in steady state. The solution is available in two ways. One is solved online, the other, offline. The results are compared with each other.

4.1 Simulation Condition The circuit and control parameters in the simulations are listed in Tables 1 and 2, respectively.

The problem setup is as follows. The load is firstly in the sleep mode. Then, received by the request of some task, the operation mode changes from the sleep mode to the active mode. After completion of the task, the mode is switched back to the idling mode, again. In such a situation, the output voltage should be kept at the reference value. The operation mode is assumed to be switched to active from sleep at $0.3[\mathrm{~ms}]$ and switched back to sleep from the active at $0.3[\mathrm{~ms}]$ later after that.

4.2 Simulation Result The partition of the state obtained by offline calculation, that is, mp-MIQP and their closeups are shown in Figs. 3 and 4, respectively. In each region of Fig. 3, the optimal input sequence is given out. The figures of state partition as shown in Figs. 3 and 4 are generated by means of Multi-Parametric Toolbox ${ }^{(10)}$. In Fig. 3, the number of state partition is limited to at most $2^{N_{p}}$. Each partition is constructed by linear inequalites. In each partition, the solution of mp-MIQP given by Eq. (43) is assigned. The state, $\left[\begin{array}{ll}i_{l} & v_{c}\end{array}\right]^{\prime}$, is judged at each control sampling. It can be simply performed since the obtained state partition

Table 1. Circuit Parameters

\begin{tabular}{|c|c|}
\hline source voltage $v_{s}$ & $5[\mathrm{~V}]$ \\
\hline inductance $x_{l}$ & $2.2[\mu \mathrm{H}]$ \\
\hline internal resistance $r_{l}$ & $20[\mathrm{~m} \Omega]$ \\
\hline capacitance $x_{c}$ & $220[\mu \mathrm{F}]$ \\
\hline $\operatorname{ESR} r_{c}$ & $20[\mathrm{~m} \Omega]$ \\
\hline
\end{tabular}

Table 2. Control Parameters

\begin{tabular}{|c|c|}
\hline control period $T_{s}$ & $1[\mu \mathrm{s}]$ \\
\hline predictive horizon $N_{p}$ & 5 \\
\hline upper limit $i_{l, \max }$ & $10[\mathrm{~A}]$ \\
\hline reference value $v_{\text {ref }}$ & $2[\mathrm{~V}]$ \\
\hline simulation period & $0.9[\mathrm{~ms}]$ \\
\hline
\end{tabular}

is constructed by linear inequalites. Focus on the white region at the right bottom corner in Figs. 3 or 4 . When the state $\left[\begin{array}{ll}i_{l} & v_{c}\end{array}\right]^{\prime}$ enters the region, switch $S_{1}$ shown in Fig. 1 is turned off since the constraint about the inductor current given by Eq. (37) can be satisfied no longer.

The output voltage is shown in Fig. 5. From Fig. 5, it can be seen that the output voltage holds at the specified value in the steady state. To see the transition, the closeups of Fig. 5 and the corresponding switching signal around the mode changes are shown in Figs. 6 and 7. Fig. 6 corresponds to the mode
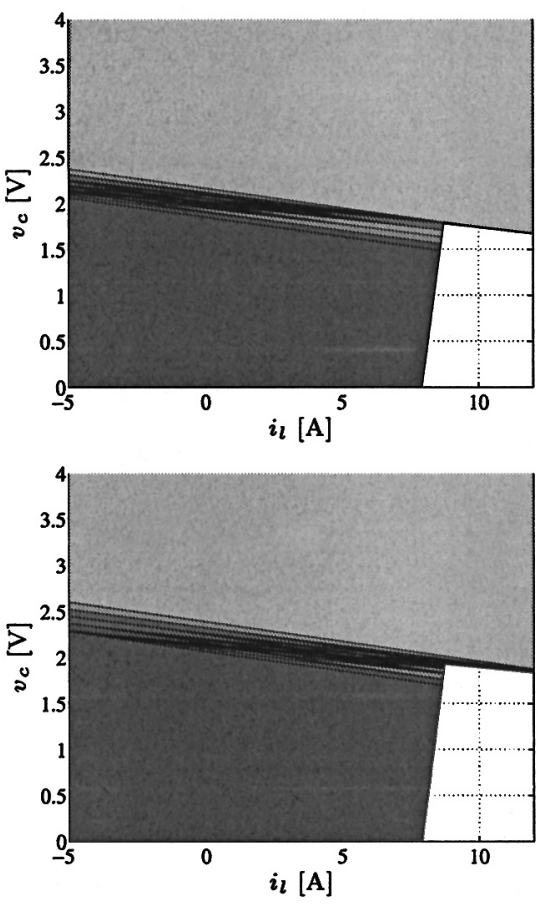

Fig. 3. State partition (top: sleep, bottom: active)
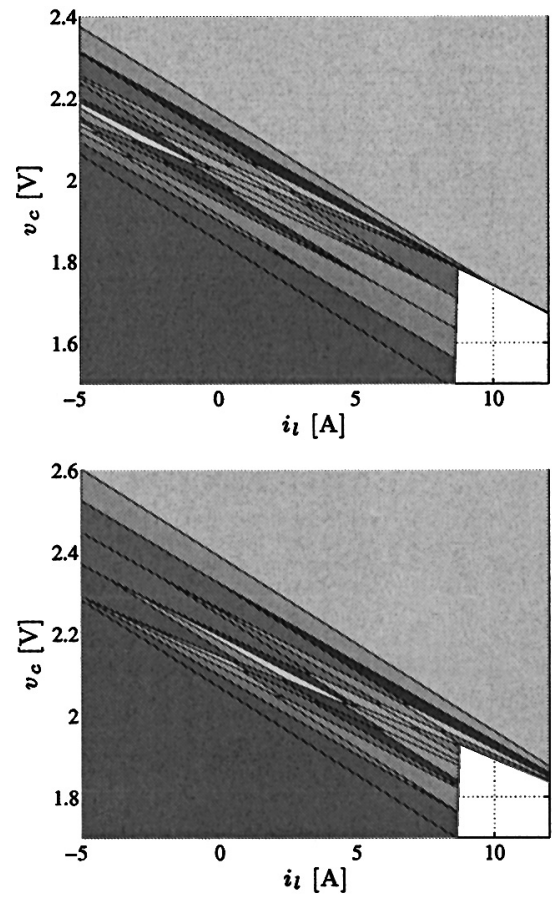

Fig. 4. State partition closeup (top: sleep, bottom: active) 

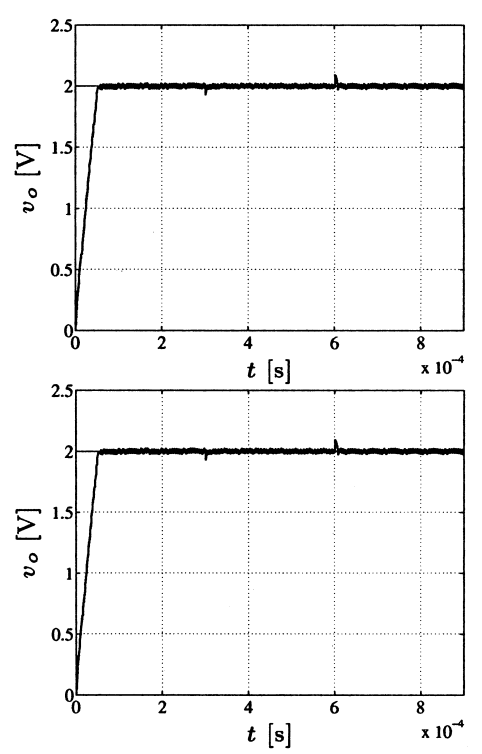

Fig. 5. Output voltage $v_{o}$ (top: MIQP, bottom: mpMIQP)
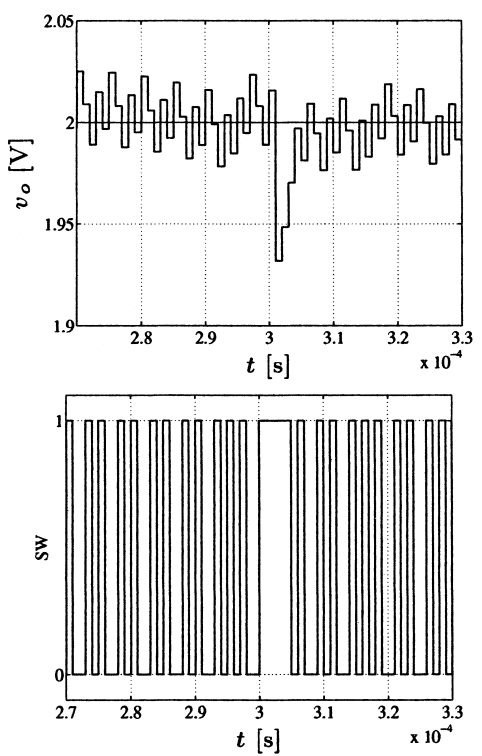

Fig. 6. Closeups of output voltage $v_{o}$ (top) and switching signal (bottom), (active from sleep)

switching from sleep to active while Fig. 7, from active to sleep. Figs. 6 and 7 illustrate that the output voltage tracks to the voltage reference quickly in spite of mode changes.

Let us turn to the inductor current, i.e. $i_{l}$ in Fig. 5, which is shown in Fig. 8. Focus on the startup in Fig. 8, then it can be seen that the inductor current is not over the limitation, 10 [A]. Let us focus on the tracking performance in the two figures in Fig. 5. It can be seen that the bottom figure in Fig. 5 is very similar to the top. The fact would hold unless modeling error and disturbance input exist although it has not been proved strictly so far. Recall that the bottom figure in Fig. 5 is given by mp-MIQP which is solved offline. Therefore, for practical point of view, the method by mp-MIQP with less computation burden is more useful than that by MIQP.

Now, there exist various parameter perturbation in electrical circuits. For the reason, in the subsequent, let us consider cases the actual values are different from those used in
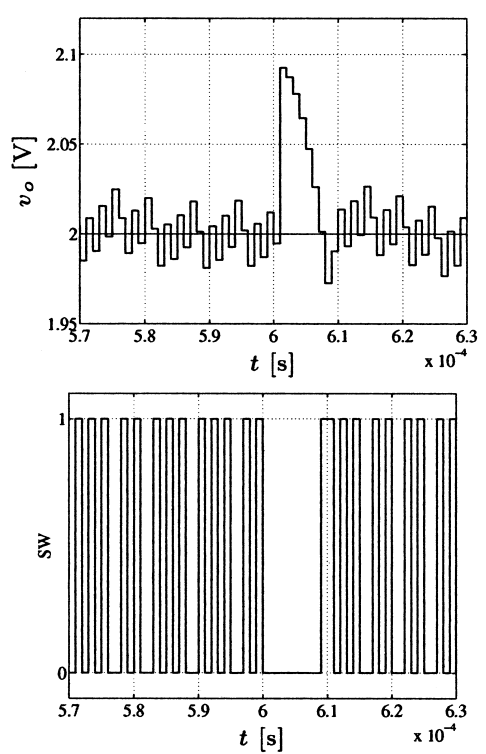

Fig. 7. Closeups of output voltage $v_{o}$ (top) and switching signal (bottom), (sleep from active)

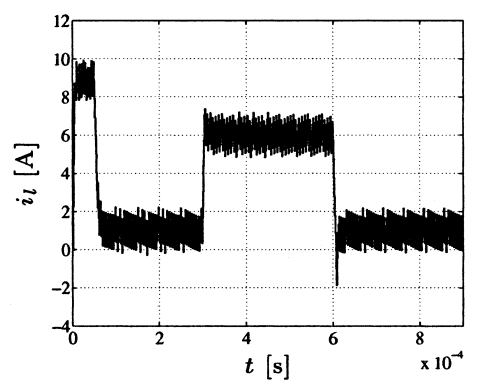

Fig. 8. Inductor current $i_{l}$

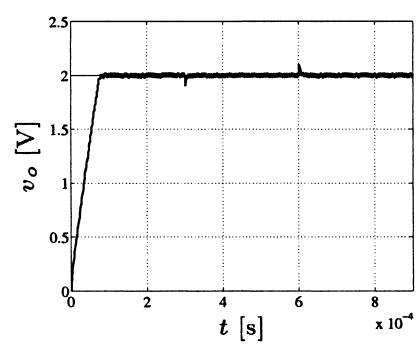

(a) nominal $x_{c}$ plus $50 \%$

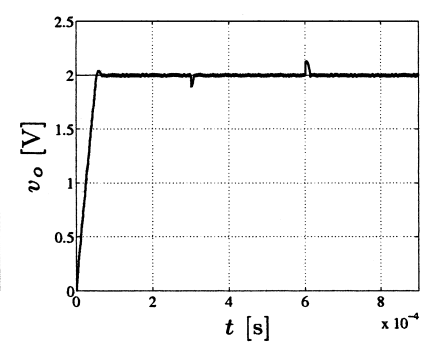

(b) nominal $x_{l}$ plus $50 \%$
Fig. 9. Output voltage $v_{o}$

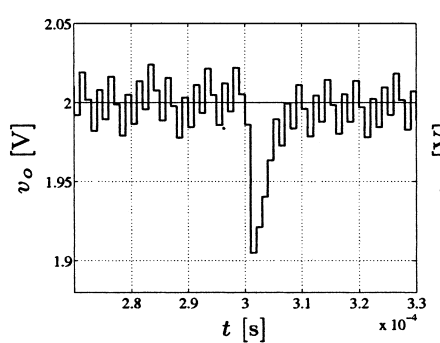

(a) nominal $x_{c}$ plus $50 \%$

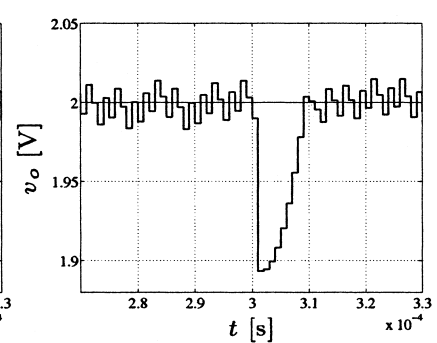

(b) nominal $x_{l}$ plus $50 \%$
Fig. 10. Output voltage $v_{o}$ closeup

the model in the proposed method. Suppose that the value of capacitance or inductance is the nominal value plus $50 \%$. Then, the output is shown in Fig. 9. Similarly, the closeups of Fig. 9 are shown in Figs. 10 and 11. From Figs. 9 to 11, the output voltage tracks to the voltage reference even if some 


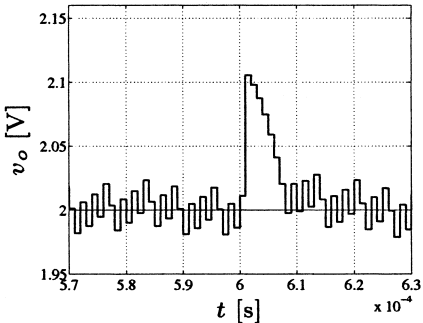

(a) nominal $x_{c}$ plus $50 \%$

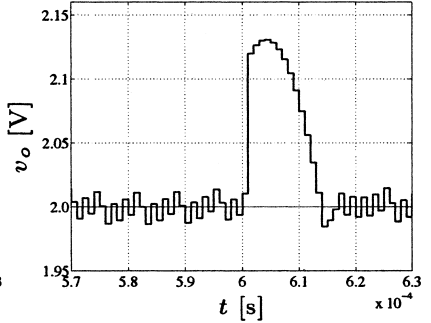

(b) nominal $x_{l}$ plus $50 \%$
Fig. 11. Output voltage $v_{o}$ closeup

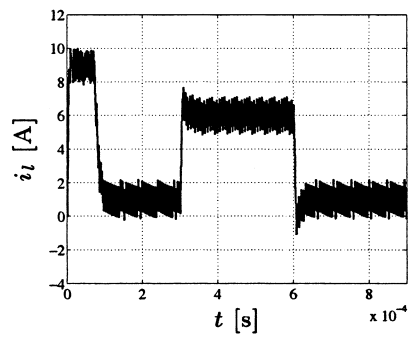

(a) nominal $x_{c}$ plus $50 \%$

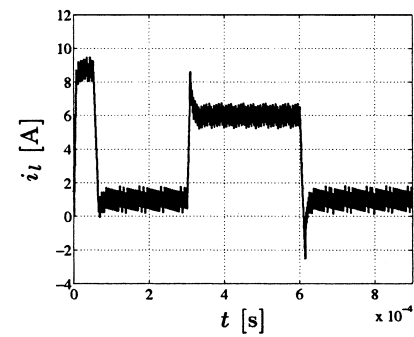

(b) nominal $x_{l}$ plus $50 \%$
Fig. 12. Inductor current $i_{l}$

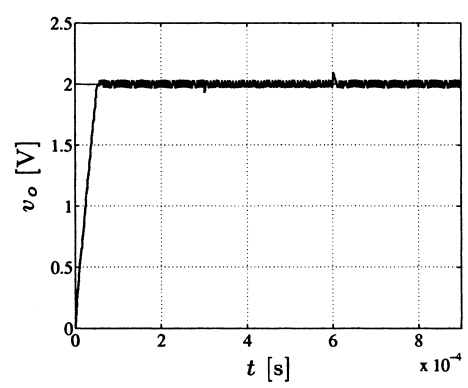

Fig. 13. Output voltage $v_{o}$

parameters are different from those used in the model. Differently from the results above, control performance is somewhat deteriorated. The reason of performance degradation is in that the predicted state is different from the actual state. However, as shown in Fig. 12(b), the current oscillation is limited since the inductance $x_{l}$ of which value is not so small smoothens it.

Next, let us consider how to reduce the switching loss. This can be achieved by adding the term about the number of switchings in the objective function of Eq. (28). In Eq. (28), $\tilde{H}$ is set as follows,

$$
\tilde{H}=\left(\Pi_{1}-\Pi_{2}\right)^{\prime} Q\left(\Pi_{1}-\Pi_{2}\right)
$$

where $\Pi_{1}, \Pi_{2}$ and $Q$ are, respectively,

$$
\begin{aligned}
& \Pi_{1}=\left[\begin{array}{ll}
0 & \\
\vdots & I_{N_{p}-1} \\
0 &
\end{array}\right] \in \mathbb{R}^{\left(N_{p}-1\right) \times N_{p}}, \\
& \Pi_{2}=\left[\begin{array}{rr} 
& 0 \\
I_{N_{p}-1} & \vdots \\
& 0
\end{array}\right] \in \mathbb{R}^{\left(N_{p}-1\right) \times N_{p}}, \\
& Q=q I_{N_{p}-1} . \cdots \ldots \ldots \ldots \ldots \ldots
\end{aligned}
$$

Note that the value of the objective function of Eq. (28) increases in response to the number of switchings. Therefore,

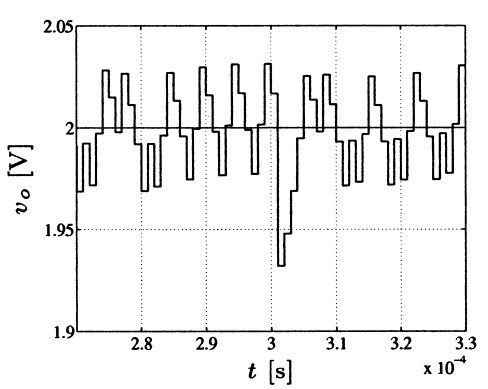

Fig. 14. Output voltage closeup $v_{o}$

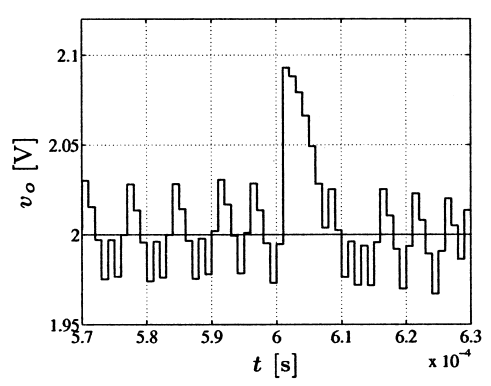

Fig. 15. Output voltage closeup $v_{o}$

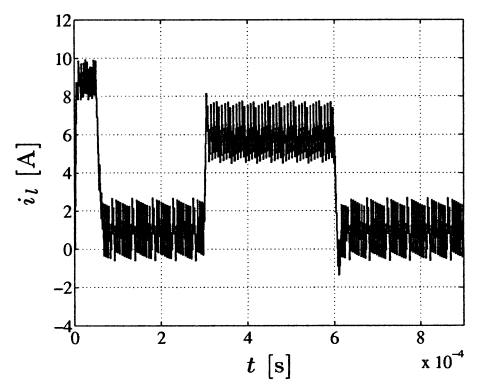

Fig. 16. Inductor current $i_{l}$

the switching loss can be reduced dependent on $q>0$ in Eq. (50). In Eq. (50), $q=10^{-3}$ is determinedby trial and error for simulation. Fig. 13 shows the output result for $\tilde{H}$ of Eq. (47). Similarly, the closeups of Fig. 13 are shown in Figs. 14 and 15. From Figs. 13 to 15, the output voltage tracks to the voltage reference even if the term to reduce switching is added in objective function. Fig. 16 also shows that the inductor current is not over the limitation, 10[A].

\section{Conclusions}

In this paper, a control method for dc-dc converter has been proposed. The dc-dc converter has been modeled as a mixed logical dynamical logic system since it has both continuous and discrete variables. For the control, a model predictive control (MPC) based method has been introduced. The optimization problem has been solved as a multi-parametric programming problem. The result has been obtained as the state space partition so that online control can be applied. As a result, the computation time was shortened without being deteriorated control performance. In addition, even if that the values used for prediction are different from those of the actual ones, the output voltage has been tracked to the voltage reference. Finally, introduction of the term to reduce the switching in the objective function, the output voltage has been tracked to the reference at the expense of tracking performance. 
Implementation of the proposed method is currently being developed. Further researches include the robustness analysis for implementation.

\section{Acknowledgment}

We are grateful to the Okasan-Kato Foundation for support to the work.

(Manuscript received Aug. 29, 2006,

revised Oct. 18, 2006)

\section{References}

( 1 ) Hybrid systems I, II, III, IV, V, Lecture Notes in Computer Science, 736, 999, 1066, 1273, 1567, New York, Springer-Verlag (1993-1998)

( 2 ) T. Ushio: "Expectations for Hybrid Systems", Systems, Control and Information, Vol.46, No.3, pp.105-109 (2002)

( 3 ) K. Asano, K. Tsuda, A. Bemporad, and M. Morari: "Predictive Control for Hybrid Systems and Its Application to Process Control", Systems, Control and Information, Vol.46, No.3, pp.110-119 (2002)

( 4 ) M. Ohshima and M. Ogawa: "Model Predictive Control-I- Basic Principle: history \& present status", Systems, Control and Information, Vol.46, No.5, pp.286-293 (2002)

( 5 ) M. Fujita and M. Ohshima: "Model Predictive Control -VI- Model Predictive Control for Hybrid Systems", Systems, Control and Information, Vol.47, No.3, pp.146-152 (2003)

( 6 ) F. Borrelli, M. Baotic, A. Bemporad, and M. Morari: "An efficient algorithm for computing the state feedback optimal control law for discrete time hybrid systems", In Proc. American Contr. Conf., pp.4717-4722 (2003)

( 7 ) A. Bemporad and M. Morari: "Control of systems integrating logic, dynamics, and constraints", Automatica, Vol.35, No.3, pp.407-427 (1999)

( 8 ) T. Geyer, G. Papafotiou, and M. Morari: "On the Optimal Control of SwitchMode DC-DC Converters", Hybrid Systems: Computation and Control, Vol.2993, pp.342-356, Lecture Notes in Computer Science (2004)

(9) G. Papafotiou, T. Geyer, and M. Morari: "Hybrid Modelling and Optimal Control of Switch-mode DC-DC Converters", IEEE Workshop on Computers in Power Electronics (COMPEL), pp.148-155, Champaign, IL, USA (2004)

(10) M. Kvasnica, P. Grieder, M. Boatić and F.J. Christophersen, "MultiParametric Toolbox (MPT)”, Institut für Automatik (2005)
Nobuhiro Asano (Student Member) born on 1983. He received the

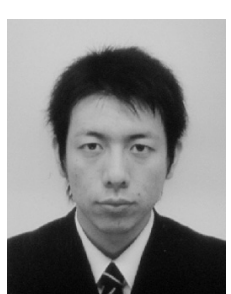
B.S. degree from Mie University in 2006. He has been a master's course candidate since 2006. His research interests are optimization control of powerelectronics circuits and motor control based on hybrid dynamical system theory.

Tadanao Zanma

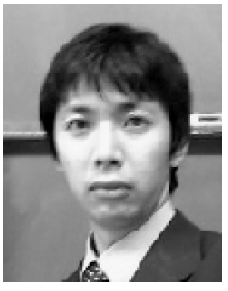

(Member) born on May 2, 1972. He received the B.S., M.S. and Dr. Eng. degrees from Nagoya University in 1995, 1997 and 2000, respectively, all in Electrical Engineering. He has been with Mie University as an Research Associate since 2000. His current research interests include hybrid dynamical control, discrete event systems, sequential control systems, especially, motion control based on mixed logical dynamical systems. He is a member of IEE, SICE, ISCIE and IEEE.

Muneaki Ishida (Member) born on April 12, 1952. He received the

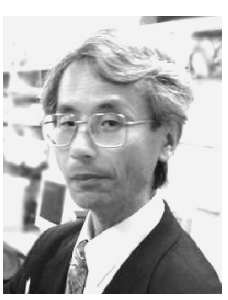
B.S., M.S. and Ph.D degrees from Nagoya University in 1975, 1977 and 1980, respectively, all in Electrical and Electronic Engineering. He was with Nagoya University as a Research Associate in the Department of Electrical Engineering from 1980 to 1987. Since 1987, he has been with Mie University, initially as an Associate professor and from October, 1996 as a Professor. He is engaged in research on static power converters, AC motor drive systems and development of new type motors using piezo-electric devices. He has received five paper awards: The Institute of Electrical Engineers thesis prizes in 1993 and 2001; The Institute of Electrical Engineers writing prize in 1999; The Society of Instrument and Control Engineers thesis prize in 2002; The Welding Society thesis prize in 2005 . He chaired many technical sessions of national/international conferences. 